\title{
Validity of clinically significant change classifications yielded by Jacobson-Truax and Hageman-Arrindell methods
}

\author{
Fiona R. Ronk ${ }^{1,2}$, Geoffrey R. Hooke ${ }^{1,2}$ and Andrew C. Page ${ }^{1 *}$
}

\begin{abstract}
Background: Reporting of the clinical significance of observed changes is recommended when publishing mental health treatment outcome studies and is increasingly used in routine outcomes monitoring systems. Since recovery rates vary with the method chosen, we investigated the validity of classifications of clinically significant change when the Jacobson-Truax method and the Hageman-Arrindell method were used.

Methods: Of 718 inpatients who completed the Depression Anxiety Stress Scales (DASS-21) and Quality of Life Enjoyment and Satisfaction Questionnaire at admission and discharge to a psychiatric clinic, 355 were invited (and 119 agreed) to complete the questionnaires and the Recovery Assessment Scale six weeks post discharge.

Results: Both the JT and HA methods showed comparably good validity when referenced against the other indices. Clinically significant change on the DASS-21 was related to a greater consumer-based sense of recovery, greater perceived quality of life, and fewer readmissions to hospital within 28 days of discharge.

Conclusions: Since there was found to be no advantage to using one method over another when recovery is of interest, the simpler JT method is recommended for routine usage.
\end{abstract}

Keywords: Clinical significance, Validity, Outcomes, Recovery, Quality of life

\section{Background}

Clinical significance categorisations aim to provide a meaningful classification of treatment outcomes [1,2]. The most widely used calculation for clinical significance is the Jacobson-Truax method $[3,4]$ which considers the reliability of the change made (Reliable Change Index; $\mathrm{RCI}$ ) in the context of the overall distribution that the patient is likely to belong to post-treatment (functional or dysfunctional). Clients moving reliably into the functional distribution are recovered. Clients have improved if they have made a reliable change but remain in the dysfunctional population, unchanged if they have not made a reliable change, and deteriorated if they have reliably worsened.

Given recommendations to report rates of clinically significant change, it is important that classifications

\footnotetext{
* Correspondence: andrew.page@uwa.edu.au

${ }^{1}$ School of Psychology, The University of Western Australia, 35 Stirling Hwy,

Crawley 6009, Australia

Full list of author information is available at the end of the article
}

are valid [5]. Supporting the ecological validity of the Jacobson-Truax (JT) method, clinically significant change on the Symptom Check List-90 Revised (SCL-90R) [6] relates to client's satisfaction with therapy [7] and client and therapist-rated change [8]. The convergent validity of classifications of clinically significant change for depressed patients across different depression measures has also been demonstrated [9]. Likewise, clients who made a clinically significant change on the Outcomes Questionnaire-45 (OQ-45) [10] also made clinically significant change on the SCL-90R, the Social Adjustment Rating Scale, the Inventory of Interpersonal Problems, and the Quality of Life Inventory [11]. Newnham, Harwood, and Page [12] determined that clinically significant change on the Medical Outcomes Short Form Questionnaire (SF-36) [13] was associated with a greater perceived quality of life, as well as greater clinician-rated functioning. Furthermore, Wise [14] demonstrated that $56 \%$ of substance abuse clients who made a reliable change on the SCL-90R had a clinically meaningful change in the 
percentage of days abstinent from substances. Ronk and colleagues [15] demonstrated that when clinical significance based on the JT method is assessed using different measures related to depression (Quality of Life Enjoyment Scale; Depression scale of the DASS-21; and SF-36 Mental Health Scale), the results are largely convergent. Therefore, these findings support the ecological validity of clinical significance classifications for the JT method of calculating the clinical significance.

\section{Potential alternatives to JT method}

However, there exist several other methods to classify the clinical significance of a treatment outcome, including the Gulliksen Lord Novick method (GLN) [16, 17], the Nunnally-Kotsch method (NK) [18], the EdwardsNunnally method (EN) [19], the Hageman-Arrindell method (HA) [20] and Hierarchical Linear Modelling (HLM) [21]. McGlinchey, Atkins, and Jacobson [22] found that the HA method classified clients significantly differently to the JT, GLN, and EN methods. The HA method was less sensitive since a greater amount of client change was required for a client to be considered reliably improved. Similarly, Ronk, Hooke, and Page [15] found that while the JT, GLN, NK, EN calculation methods yielded similar rates of clinically significant change, the HA method produced consistently distinct classifications. Therefore, the HA method is more conservative in assigning classifications of recovered to patients than other methods.

The reason for differences between the classification rates lies in the method of calculations. The JT method classifies a client's outcome based on the reliability of the pre- to post-treatment change and whether or not the client has moved from the dysfunctional population to the functional population. The calculation uses clients' observed scores, which contain measurement error. The HA method attempts to correct regression to the mean by using an approximation of true scores rather than observed scores. In addition, while the JT method uses a cut-off score to separate the functional and dysfunctional distributions, the HA method uses a cut-off index score which allows users to determine that a client has passed the cut-off score in the correct direction with $95 \%$ confidence.

These differences between rates of recovery based on the JT and HA methods of classifying change need to be explored. However, as previously stated by Hsu [23], one method cannot be recommended over another based purely on higher or lower rates of classifications of clinically significant change. It is important to determine whether one method's recovered clients experience changes in other areas of importance, such as quality of life, that reasonably correspond with the concept of recovery, when compared to other classification methods (see [22]). Thus, analyses will only be conducted using the JT and HA methods, since the remaining three methods explored in McGlinchey et al., [22] and Ronk et al. [15] were largely similar to the JT method.

\section{Recovery evaluation}

While in clinical research symptom reduction is often synonymous with recovery, 'consumer-based recovery' captures the notion that there are many facets to recovery, including hope, healing, empowerment, self-identity, pursuing meaningful goals, developing connections with others, and having a sense of control [24, 25]. This definition of recovery posits that a focus on symptom reduction alone is too narrow, as clients who report severe symptoms can still experience improvements in other aspects of their lives [26, 27] and vice versa. Therefore, in the present study the first recovery evaluation variable is a consumer-based measure of recovery; the Recovery Assessment Scale (RAS) [28].

Another domain associated with the consumer-based conceptualisation of recovery is quality of life [29]. Although symptoms and quality of life are not mirror images on one another, an increase in symptoms relates to a decrease in quality of life for patients with Major Depressive Disorder [30]. If the inverse of this is also true, and clinically significant change on a symptom measure relates to significant improvements in perceived quality of life, then this will provide convergent validity for classifications of the recovered category. Therefore, the second recovery evaluation measure chosen is the Quality of Life Enjoyment and Satisfaction Questionnaire (Q-LES-Q) [31, 32].

In addition, it is important to include an objective indicator of treatment outcome. Whether or not a patient has been readmitted to an inpatient facility soon after their discharge has a logical relationship to outcome. Readmission to hospital, specifically within the 28 day period following discharge, is used as a national clinical indicator of the quality of care [33]. Readmission to hospital within 28 days is considered a poor outcome associated with more severe symptoms [34, 35], and prior hospital admissions [36]. Specifically, being readmitted to hospital within 28 days is not consistent with that person being considered as recovered $[37,38]$.

\section{Current study}

The focus of the current study is on the categorisation of patients as recovered, which is defined according to statistical methods for reporting clinical significance as both (a) making a statistically reliable change during treatment; and (b) belonging to the 'functional' population at post-treatment. Firstly, we aim to examine the validity of the JT method for assessing clinically significant change by exploring the relationship between classifications of recovered and three variables related to the concept of recovery. It is necessary to explore the links between clinical 
significance classifications and these criterion measures before any further assumptions can be made about the validity of the clinical significance methodology. It is hypothesised that those patients who are classified as recovered by either the JT or HA methods, when compared to those who are not classified as recovered, will:

(a) score higher on the Recovery Assessment Scale (RAS) $[39,40]$ indicative of a greater sense of consumer-based recovery;

(b)score higher on the Quality of Life Enjoyment and Satisfaction Questionnaire (Q-LES-Q) [31], indicative of a greater perceived enjoyment and satisfaction with life; and

(c) have lower rates of readmission to hospital within 28 days of their discharge, indicative of a more successful post-discharge period.

Secondly, if there is an association between a clinically significant change and our three criterion measures, we aim to determine whether one method demonstrates more convergent validity than another.

\section{Method}

\section{Participants and procedure}

Participants were 718 consecutively admitted patients with complete data discharged from a private psychiatric hospital between April 2011 and January 2012. The mean age was 42.9 years $(S D=15.1)$ and the mean length of hospital stay was 17.4 days $(S D=14.8)$. Married patients made up $50.3 \%$ of the sample, $33.2 \%$ were single, and $16.5 \%$ were separated, divorced, or widowed. Participants were given diagnoses by their treating psychiatrist. The sample consisted of patients with primary diagnoses of mood (56.1\%), anxiety (19.4\%), substance use (13.7\%), and psychotic disorders (5.9 \%) as well as other diagnoses (4.9\%).

While in hospital, patients complete a range of group therapies led by clinical psychologists and occupational therapists including cognitive behavioural therapy, interpersonal therapy, and structured activity-based therapy while under the care of nursing staff and their psychiatrist. As part of routine quality assurance at the hospital, patients were invited to complete questionnaire measures at both admission and discharge. Participants provided informed consent and the study had ethical approval (\#2557).

Patients completed the DASS-21 and Q-LES-Q at admission and discharge. A total of 718 patients discharged during the period from April 2011 and January 2012 completed both measures at admission and at discharge. These data were used to assess clinical significance of change from pre- to post-treatment as measured by the DASS-21 as well as quality of life score at discharge and readmission to hospital within 28 days of discharge. A cohort of 355 patients discharged during the first half of the study period was invited to complete the DASS-21 and RAS six weeks after discharge. The total response rate was $41.1 \%$, which compares favourably with other mail-out surveys. Only cases with complete data $(n=119)$ were used in the analyses of scores six weeks postdischarge. Age differed significantly between responders $(M=48.0$ years; $S D=15.7)$ and non-responders $(M=$ 40.4 years; $S D=15.0) ; t(353)=4.68, p<.05$. Length of stay in hospital was longer $(M=19.2$ days; $S D=15.8)$ for patients who responded compared to patients who did not respond $(M=16.1$ days; $S D=12.2) ; t(353)=2.16, p<.05$. There were no significant differences between responders and non-responders in symptom severity at admission or discharge and prior admissions to hospital.

\section{Measures \\ Recovery Assessment Scale (RAS)}

Scores on the RAS have high internal consistency $(\alpha=.93)$ and test-retest reliability $(\alpha=.88)[28,41]$. The validity of RAS score interpretations demonstrates convergent validity $[28,42]$ with correlations with other recovery-oriented scales ranging from $r=.20-.68$. The RAS demonstrates divergent validity from symptom or function-based measures such as the Health of the Nation Outcome Scales (HoNOS) [43, 44]. The RAS originally had 41 items, however Hancock et al. [40] removed 10 items due to poor fit statistics or item redundancy, resulting in a 31 -item scale that map more closely to processes associated with consumer based recovery in the literature (e.g., symptom management, a sense of control). Based on results of the Rasch analysis [40], the 31-item version of the RAS with a five-point rating scale ( $1=$ strongly disagree; $5=$ strongly agree) was chosen to determine recovery scores in the current study. Scores are summed to form one score representing 'recovery', with a minimum possible score of 31 and a maximum possible score of 155 . A higher score indicates a stronger experience of recovery.

\section{Depression Anxiety Stress Scales 21 (DASS-21)}

Lovibond and Lovibond [45] the DASS-21 measures levels of depression, anxiety, and stress. Respondents rate 21 items such as "I felt down-hearted and blue" and "I felt that life was meaningless" on a scale ranging from zero to three. Within each scale, the total score is doubled so that the minimum score is zero and the maximum score is 42 . The scores on each scale have high internal consistency ( $\alpha=.88$ for Depression; $\alpha=.82$ for Anxiety and $\alpha=.90$ for Stress; [46]) and the interpretations of the construct demonstrate good convergent and discriminant validity [45-48]. 


\section{Quality of Life Enjoyment and Satisfaction Questionnaire- Short Form (Q-LES-Q)}

Endicott et al. [31] the Q-LES-Q is a 14 item self-report scale assessing quality of life across domains such as physical health, and household activities. Respondents rate their satisfaction with each domain on a 5-point scale. Item scores are added and transformed onto a scale ranging from 0 to 100, with higher scores indicative of higher perceived quality of life. Scores on the Q-LES-Q demonstrate high internal consistency $(>.90)$ and test-retest reliability (.63-.89), and the interpretations of the scores show good construct validity [30, 32, 49].

\section{Clinical significance calculation methods}

The current study used the Jacobson-Truax method of clinical significance classification [3, 4] and the Hageman Arrindell method of clinical significance classification [19]. When using the JT the cut-off between the dysfunctional population and the functional population can be calculated using one of three formulas. The present study used cut-off ' $\mathrm{C}$ ' to represent the cut-off between the functional and dysfunctional population as recommended by Hsu [50]. In addition to classifications of clinical significance made using scores from the time of pre-treatment to post-treatment, classifications were also calculated using pre-treatment scores and scores at six weeks post-treatment.

\section{Data analysis}

Independent sample $t$-tests and corresponding measure of effect size, Cohen's $d$, will be used to evaluate the difference in RAS scores and quality of life scores for those patients who make a clinically significant change on the DASS-21 and those who do not. Chi-squared analysis $\left(\chi^{2}\right)$ and corresponding measure of effect size, phi $(\phi)$ will be used to assess the difference in readmission rates within 28 days between those patients who make a clinically significant change on the DASS-21 and those who do not. Following Cohen [51], the conventions for small, medium and large effect sizes are respectively .20, .50 and .80 for Cohen's d, and $.10, .30$ and .50 for $\Phi$ (and the Pearson correlation coefficient, $r$ ).

\section{Results}

Scores for each scale of the DASS-21 decreased between pre-treatment and post-treatment, and quality of life increased (Table 1). DASS-21 scales at pre-treatment, post-treatment, and between change scores were moderately inter-correlated. Pre-treatment correlations were significant $(p<.01) ; r_{(\text {Dep }} \&$ Anx $)=.52, r_{(\text {Dep }} \&$ Str $)=.57$, and $r_{\text {(Anx }} \&$ Str) $=.69$. Post-treatment correlations were significant $(p<.01) ; r_{(\text {Dep }} \&$ Anx $)=.70, r_{(\text {Dep }} \&$ Str $)=.78$, and $\left.r_{(\mathrm{Anx}} \& \mathrm{Str}\right)=.74$. Correlations between change scores were also significant $(p<.01) ; r_{\text {(Dep }} \&$ Anx $)=.59$, $r_{\text {(Dep }} \&_{\text {Str) }}=.68$, and $r_{\text {(Anx }} \&$ Str) $=.69$.

When patients are classified using the JT method, there are higher rates of clinically significant change than when the HA method is used (Table 2). This suggests that the HA method is more stringent in its classification of recovery. Classifications of deterioration yield identical proportions with both methods. Of the 718 patients discharged, 64 (8.9 \%) were readmitted within 28 days of their discharge from hospital.

\section{Recovery assessment scale}

Of 119 patients who completed the RAS six weeks following discharge from hospital (Table 3), between 18.5 and $56.3 \%$ of patients were classified as recovered, depending on which DASS-21 scale a patient was measured on and with which clinical significance calculation method. Patients who were classified as recovered on each scale of the DASS-21 according to both the JT and HA methods scored significantly higher on the RAS than those who made no clinically significant change. A similar pattern was found when patients who were classified as recovered according to the HA method were compared with those who were not. This suggests that both the JT and HA methods for evaluating clinically significant change (i.e., a classification of recovered) demonstrate construct validity, as clinically significant change on a symptom measure, the DASS-21, is related to higher scores on the RAS, representative of a more positive perception of 'consumer-based' recovery.

Table 1 Means and standard deviations (in parentheses) for the DASS-21, Q-LES-Q and RAS at pre-treatment, post-treatment, and six weeks post-treatment

\begin{tabular}{lllllll}
\hline & & \multicolumn{2}{l}{ DASS-21 scale } & & Q-LES-Q & RAS \\
\cline { 2 - 4 } & $n$ & Depression & Anxiety & Stress & & \\
\hline Pre-treatment & 718 & $28.27(11.84)$ & $20.53(10.85)$ & $26.96(10.24)$ & $32.59(17.42)$ & - \\
Post-treatment & 718 & $12.82(10.79)$ & $10.18(8.95)$ & $13.63(9.69)$ & $52.97(18.69)$ & - \\
Six weeks post-treatment & 119 & $12.66(11.69)$ & $9.78(9.16)$ & $14.13(10.53)$ & - & $119.15(18.26)$ \\
Range of measure & & $0-42^{\mathrm{a}}$ & $0-42^{\mathrm{a}}$ & $0-42^{\mathrm{a}}$ & $0-100^{\mathrm{b}}$ & $31-155^{\mathrm{b}}$ \\
\hline
\end{tabular}

DASS-21 depression anxiety stress scales - 21, Q-LES-Q quality of life enjoyment and satisfaction questionnaire, RAS recovery assessment scale

ahigher scores reflect more negative functioning. ${ }^{b}$ higher scores reflect more positive functioning 
Table 2 Percentage of patients classified into each clinical significance category by the Jacobson-Truax (JT) method and the Hageman-Arrindell (HA) method based on DASS-21 scale scores calculated across two time periods

\begin{tabular}{|c|c|c|c|c|c|c|}
\hline & \multicolumn{2}{|c|}{ DASS-21 Depression scale } & \multicolumn{2}{|c|}{ DASS-21 Anxiety scale } & \multicolumn{2}{|c|}{ DASS-21 Stress scale } \\
\hline & JT method & HA method & JT method & HA method & JT method & HA method \\
\hline \multicolumn{7}{|c|}{ Pre-treatment to post-treatment $(n=718)$} \\
\hline Recovered & $57.5 \%$ & $42.1 \%$ & $36.8 \%$ & $19.1 \%$ & $58.9 \%$ & $46.7 \%$ \\
\hline Improved & $14.9 \%$ & $35.9 \%$ & $14.6 \%$ & $48.1 \%$ & $11.6 \%$ & $29.9 \%$ \\
\hline Unchanged & $25.5 \%$ & $19.9 \%$ & $46.4 \%$ & $31.3 \%$ & $27.1 \%$ & $21.0 \%$ \\
\hline Deteriorated & $2.1 \%$ & $2.1 \%$ & $2.2 \%$ & $1.5 \%$ & $2.4 \%$ & $2.4 \%$ \\
\hline \multicolumn{7}{|c|}{ Pre-treatment to six weeks post-treatment $(n=119)$} \\
\hline Recovered & $49.5 \%$ & $37.8 \%$ & $36.1 \%$ & $18.5 \%$ & $56.3 \%$ & $47.9 \%$ \\
\hline Improved & $11.8 \%$ & $29.4 \%$ & $14.3 \%$ & $47.0 \%$ & $10.1 \%$ & $23.5 \%$ \\
\hline Unchanged & $34.5 \%$ & $28.6 \%$ & $47.9 \%$ & $32.8 \%$ & $23.5 \%$ & $18.5 \%$ \\
\hline Deteriorated & $4.2 \%$ & $4.2 \%$ & $1.7 \%$ & $1.7 \%$ & $10.1 \%$ & $10.1 \%$ \\
\hline
\end{tabular}

\section{Quality of life}

Of 718 patients who completed the Q-LES-Q at posttreatment (Table 4), between 19.1 and $58.9 \%$ were classified as having achieved a clinically significant change, depending on which DASS-21 scale they were assessed on and clinical significance classification method. Perceived quality of life was greater for patients classified as recovered by the JT and HA methods than those who were not. These findings support the construct validity of clinically significant change as calculated by both the methods.

\section{Readmission to hospital within $\mathbf{2 8}$ days of discharge}

A significantly higher proportion of patients who were not considered recovered at discharge were readmitted within 28 days than those who were considered recovered by the JT method with the Depression scale $\left(\chi^{2}(1)=9.80, p=.002\right.$, $\phi=.117$; Fig. 1), the HA method with the Depression scale $\left(\chi^{2}(1)=6.93, p=.008, \phi=.098\right)$, and the HA method with the Stress scale $\left(\chi^{2}(1)=4.259, p=.039, \phi=.077\right)$. The remaining classification methods yielded no significant differences between readmission rates for recovered compared to non-recovered patients. Since patients who were not considered to have made a clinically significant change on the Depression scale were approximately twice as likely to be readmitted within 28 days of discharge than those patients whose change had been considered clinically significant, this provides support for the construct validity of recovery as evaluated by both calculation methods but only when classifications are based on certain DASS-21 scores.

\section{Discussion}

When patients who received a classification of recovered at post-treatment (calculated using the JT method with DASS-21 scores) were compared to those who were not considered recovered, the recovered patients had significantly higher RAS scores, indicative of a more positive consumer-based sense of recovery, and significantly higher Q-LES-Q scores, indicative of a greater perception of life enjoyment and satisfaction. The rate of hospital readmission within 28 days of discharge was significantly lower for those considered recovered according to the JT method with the Depression scale, and the HA method with the Depression and Stress scales. These findings provide

Table 3 Descriptive statistics for patient scores on the recovery assessment scale who have been classified as recovered or not recovered based on each DASS-21 scale using the JT and HA calculation methods

\begin{tabular}{|c|c|c|c|c|c|c|}
\hline & \multicolumn{3}{|c|}{ Classifications based on JT method } & \multicolumn{3}{|c|}{ Classifications based on HA method } \\
\hline & Depression & Anxiety & Stress & Depression & Anxiety & Stress \\
\hline \multicolumn{7}{|c|}{ Classified as recovered } \\
\hline$n$ & 59 & 43 & 67 & 45 & 22 & 57 \\
\hline Mean & 126.79 & 124.65 & 125.42 & 130.10 & 131.77 & 126.12 \\
\hline SD & 14.24 & 17.52 & 13.97 & 13.90 & 17.34 & 14.59 \\
\hline \multicolumn{7}{|c|}{ Not classified as recovered } \\
\hline$n$ & 60 & 76 & 52 & 74 & 97 & 62 \\
\hline Mean & 111.65 & 116.04 & 111.08 & 112.50 & 116.29 & 112.75 \\
\hline SD & 18.76 & 18.04 & 20.02 & 17.42 & 17.29 & 19.04 \\
\hline
\end{tabular}


Table 4 Descriptive statistics for patient scores on the quality of life enjoyment and satisfaction scale who have been classified as "Recovered" or "Not Recovered" based on each DASS-21 scale using the JT and HA calculation methods

\begin{tabular}{|c|c|c|c|c|c|c|}
\hline & \multicolumn{3}{|c|}{ Classifications based on JT method } & \multicolumn{3}{|c|}{ Classifications based on HA method } \\
\hline & Depression & Anxiety & Stress & Depression & Anxiety & Stress \\
\hline \multicolumn{7}{|c|}{ Classified as "recovered" } \\
\hline$n$ & 413 & 264 & 423 & 302 & 137 & 335 \\
\hline Mean & 58.84 & 60.79 & 58.65 & 63.03 & 66.28 & 61.28 \\
\hline SD & 15.73 & 16.94 & 16.23 & 14.87 & 16.11 & 15.53 \\
\hline \multicolumn{7}{|c|}{ Not classified as "recovered" } \\
\hline$n$ & 305 & 454 & 295 & 416 & 581 & 383 \\
\hline Mean & 45.03 & 48.43 & 44.84 & 45.68 & 49.84 & 45.71 \\
\hline SD & 19.46 & 18.16 & 19.01 & 17.78 & 17.87 & 18.20 \\
\hline
\end{tabular}

further support that classifying patients as recovered according to the Jacobson-Truax method of clinical significance calculation has construct validity when used with a symptom measure.

Despite the differences in recovery rates between the more lenient, popular JT method and the more conservative, less commonly used HA method [15, 22], a comparison of effect sizes did not uncover any significant differences between the methods. This suggests there are no meaningful differences between how the methods capture the construct of recovery as conceptualised by the variables chosen in the current study. Therefore, we echo the recommendation [1] that the JT method continue to be used since it is the most commonly used and simplest to calculate.

\section{Limitations}

It could be argued that since the current study was correlational in nature, it was not possible to determine which method was better 'calibrated' towards recovery. This is true, however the issue of calibration is an arbitrary one, since the category of recovered has demonstrated meaning from the perspective of both the patient and treatment provider. Whether the 'true' rate of recovered patients is indeed higher or lower than that determined by the JT method is not relevant if the arbitrary categories have meaning.

Although we can conclude here the JT and HA methods appear to have similar conceptualisations of the category of recovered, the current study does not allow for any comment about the validity of the categories of improved, unchanged, or deteriorated. Further research is required to determine the relationship between belonging in each of these categories and scores on relevant behavioural or functional indices, as well as individual client factors. For example, it may be that clients who are unchanged during treatment have lower scores on readiness to change measures. If this is the case, then clinicians could employ specific techniques such as motivational interviewing [52] for those clients who score low on a readiness to change measure at pre-treatment, to increase their chances of making a reliable or clinically significant change during treatment.

Of particular concern to clinicians are those people who deteriorate during treatment. Validity studies need to focus on these clients, as they are not often included

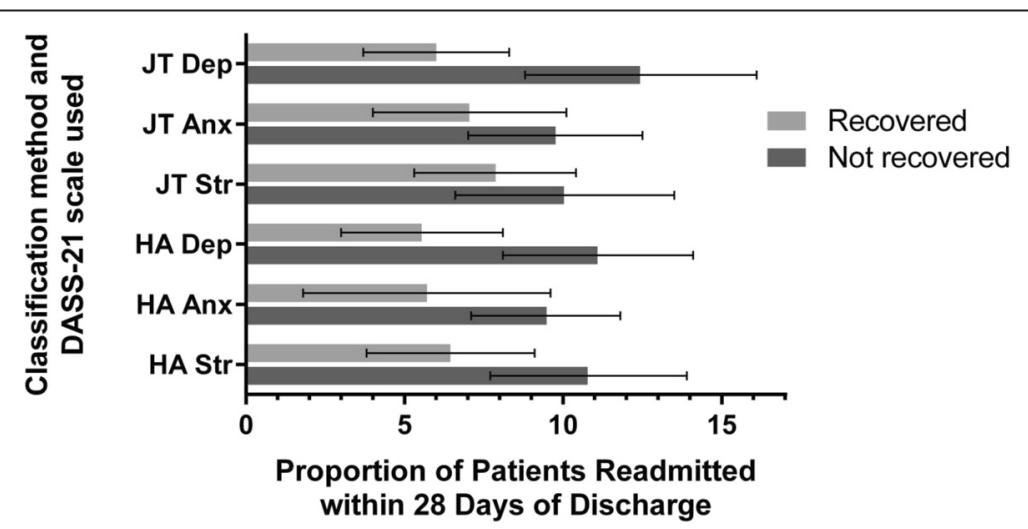

Fig. 1 Proportion of patients readmitted within 28 days of discharge who are considered recovered and not recovered by the JT and HA methods used with the DASS-21 scale scores. Error bars represent $95 \%$ confidence intervals 
in assessments of clinical significance. One reason for their lack of inclusion in such research may be the typically low proportion of clients who receive this classification. Of course, having very few deteriorators in a sample is desirable from a clinical perspective, but makes it more difficult to explore the correlates of deterioration, as in the current study. Since the present sample consisted of inpatients that generally score high on symptom measures, the chances of increasing symptoms enough to achieve a reliable deterioration are lower than in outpatient samples. An added complexity in regards to deteriorators is that they are not a homogenous group; the negative, reliable change required to be classified as deteriorated can occur anywhere along the range of the outcome measure. For example, a deterioration based on movement from the normal range to the mild range is qualitatively different to a deterioration based on movement from the severe range to the extremely severe range of a symptom measure. It therefore follows that correlates of deterioration may be equally as heterogeneous. Larger samples of patients are required to meaningfully explore the correlates of this form of patient change. Methods employed in the feedback literature [53-56] could then be used to predict which patients are "at-risk" of deteriorating, allowing clinicians to intervene during treatment. In addition to these concerns, it is relevant to note that it is not always possible or practical to calculate clinical significance. That is, some scales do not (and sometimes cannot) have relevant normative information and reliability estimates and for low prevalence mental health conditions the case for applicability needs to be made. Likewise, while the present paper has explored to some degree what is perceived as 'clinically significant change,' it is possible that the classification may vary depending on the perspective of the rater (i.e., client, clinician, carer, service provider, etc).

The use of readmission to hospital within 28 days of discharge as an index of recovery has limitations. A small proportion (5-8\%) of patients who are classified as recovered are readmitted to hospital within 28 days, and not all patients who worsen (and perhaps require readmission) will be readmitted. Furthermore, patients who require further treatment do not always require this for the same reasons as a prior admission, nor do they always seek it from the same facility. Despite this, evaluating readmission is an objective, routinely used clinical indicator of the quality of an episode of mental health care that can provide useful information. McGlinchey et al. [22] stated that if clinical significance classifications are valid, then they should mean something in practical terms, regarding whether an individual will remain recovered over time. In the current sample, although the rates of readmission were lower for patients classified as recovered than for those who were not, being assigned this classification did not remove the possibility of readmission altogether. Future research should explore the factors associated with hospital readmission subsequent to making a clinically significant change during the initial admission.

Since participants in the current study had diagnoses predominantly of mood and anxiety disorders, the current findings should generalise well to most psychiatric populations. However, for populations with mood and anxiety disorders, scores derived from self-report measures (e.g., Q-LES-Q) may be influenced by patients' current mood, their level of insight, or recent life events [57]. This issue is present in all self-report studies in psychiatric samples, and relates also to the symptom measures on which clinically significant change is measured. Furthermore, the treatment provided to patients in the current study was voluntary within an inpatient setting, therefore further research may be required to explore whether the validity of clinical significance classifications is supported in those populations where treatment is involuntary, or provided in outpatient settings. Finally, the patients who responded in the current study were older and had longer lengths of stay than those who did not respond; several hypotheses could explain this difference. However, since a focus of the study was upon the comparison of two methods of calculating clinical significance, the differences between respondents and non-respondents were not considered relevant; the more important issue was that the same patients were included in each comparison analysis.

\section{Conclusions}

Classifying change into valid clinical significance categories following mental health treatment allows treatment providers to evaluate treatment effectiveness, provide valid feedback, and allows for ongoing quality improvement. Current findings suggest that classifications of clinically significant change made using the DASS-21 demonstrate ecological, construct validity, since classifications of recovered align with more positive perceptions of consumerbased recovery, greater perceived life enjoyment and satisfaction, and a lower chance of being readmitted to hospital with 28 days of discharge. These results together with validity findings in the extant literature suggest that the commonly used Jacobson-Truax method of classifying clinically significant change does exhibit validity, and therefore the recommendation that clinical significance classifications are reported in every outcome study is warranted. Additionally, there was no discernible advantage to using the HA method over the JT method, therefore the use of the simpler, JT method, is recommended. The JT methodology provides an easy, fast, and most importantly, ecologically valid way to approximate the meaningfulness of a clients' change. 


\section{Abbreviations}

(Q-LES-Q), quality of life enjoyment and satisfaction questionnaire; DASS-21, depression anxiety stress scales; EN, Edwards-Nunnally method; GLN, Gulliksen Lord Novick method; HA, Hageman-Arrindell method; HLM, hierarchical linear modelling; HoNOS, health of the nation outcome scales; JT, Jacobson-Truax method; NK, Nunnally-Kotsch method; OQ-45, outcomes questionnaire-45; RAS, recovery assessment scale; $\mathrm{RCl}$, reliable change index; SCL-90R, symptom check list-90 revised; SF-36, medical outcomes short form questionnaire

\section{Acknowledgements}

The authors are grateful to the assistance of Moira Munro.

\section{Funding}

Funding for this research was in part by an Australian Research Council grant LP100200749.

\section{Availability of data and materials}

Data are not to be shared as they contain patient data.

\section{Authors' contributions}

FRR conducted analyses and prepared the initial drafts of the manuscript under the supervision of GRH and ACP. FRR, GRH and ACP were involved in the study design, data collection, and manuscript preparation and revision. All authors approved the final version of the manuscript

\section{Competing interests}

Authors do not have financial or non-financial competing interests to declare.

\section{Consent for publication}

Not applicable.

\section{Ethics approval and consent to participate}

Participants provided informed consent and the study had UWA Human Research and Ethics Office approval (\#2557).

\section{Author details}

'School of Psychology, The University of Western Australia, 35 Stirling Hwy, Crawley 6009, Australia. ${ }^{2}$ Perth Clinic, Perth, Western Australia.

\section{Received: 19 November 2015 Accepted: 31 May 2016}

Published online: 06 June 2016

\section{References}

1. Lambert MJ, Ogles BM. Using clinical significance in psychotherapy outcome research: the need for a common procedure and validity data. Psychoth Res. 2009;19:493-501.

2. Newnham EA, Page AC. Bridging the gap between best evidence and best practice in mental health. Clin Psychol Rev. 2011;30:127-42.

3. Jacobson NS, Follette WC, Revenstorf D. Psychotherapy outcome research: methods for reporting variability and evaluating clinical significance. Behav Ther. 1994;15:336-52

4. Jacobson NS, Truax P. Clinical significance: a statistical approach to defining meaningful change in psychotherapy research. J Cons Clin Psychol. 1991;59:12-9.

5. Schmuckler MA. What is ecological validity? A dimensional analysis. Infancy. 2001;2:419-36

6. Derogatis LB, Cleary PA. Confirmation of the dimensional structure of the SCL-90: a study in construct validation. J Clin Psychol. 1977:3:981-9.

7. Ankuta GY, Abeles N. Client satisfaction, clinical significance, and meaningfu change in psychotherapy. Prof Psychol-Res Pr. 1993;24:70-4.

8. Lunnen KM, Ogles BM. A multiperspective, multivariable evaluation of reliable change. J Consult Clin Psychol. 1998:66:400-10.

9. Ogles BM, Lambert MJ, Sawyer JD. Clinical significance of the national institute of mental health treatment of depression collaborative research program data. J Consult Clin Psychol. 1995:63:321-6.

10. Lambert MJ, Okiishi JC, Finch AE, Johnson LD. Outcome assessment: from conceptualization to implementation. Prof Psychol-Res Pr. 1998;1998:29.

11. Beckstead DJ, Hatch AL, Lambert MJ, Eggett DL, Goates MK, Vermeersch DA Clinical significance of the outcome questionnaire (OQ-45.2). Behav Analyst Today. 2003;4:86-96
12. Newnham EA, Harwood KE, Page AC. Evaluating the clinical significance of responses by psychiatric inpatients to the mental health subscales of the SF-36. J Affective Disorders. 2007;98:91-7.

13. Brazier JE, Harper R, Jones NM, O'Cathain A, Thomas KJ, Usherwood T, Westlake L. Validating the SF-36 health survey questionnaire: new outcome measure for primary care. BMJ. 1992;05(6846):160-4.

14. Wise EA. Evidence-based effectiveness of a private practice intensive outpatient program with dual diagnosis patients. J Dual Diagnosis. 2010;6:25-45.

15. Ronk FR, Hooke GR, Page AC. How consistent are clinical significance classifications when calculation methods and outcome measures differ? Clin Psychol-Sci Pr. 2012;19:167-79.

16. Hsu LM. Reliable changes in psychotherapy: taking into account regression toward the mean. Behav Assess. 1989:11:459-67.

17. Hsu LM. Regression toward the mean associated with measurement error and the identification of improvement and deterioration in psychotherapy. J Consult Clin Psychol. 1995;63:141-4.

18. Nunnally JC, Kotsch WE. Studies of individual subjects: logic and methods of analysis. Br J Clin Psychol. 1983;22:83-93.

19. Hageman WJ, Arrindell WA. Establishing clinically significant change: increment of precision between individual and group level of analysis. Behav Res Ther. 1999:37:1169-93.

20. Speer DC. Clinically significant change: Jacobson and Truax (1991) revisited. J Consult Clin Psychol. 1992;60:402-8.

21. Bryk AS, Raudenbush SW. Hierarchical linear models: applications and data analysis methods. Newbury Park: Sage; 1992.

22. McGlinchey JB, Atkins DC, Jacobson NS. Clinical significance methods: Which one to use and how useful are they? Behav Ther. 2002;33:529-50.

23. Hsu LM. Caveats concerning comparisons of change rates obtained with five methods of identifying significant client changes: comment on Speer and Greenbaum (1995). J Consult Clin Psychol. 1999;67:594-8.

24. Clarke SP, Oades LG, Crowe TP, Caputi P, Deane FP. The role of symptom distress and goal attainment in promoting aspects of psychological recovery for consumers with enduring mental illness. J Ment Health. 2009;18:389-97.

25. Jacobson NA, Greenley D. What is recovery? A conceptual model and explication. Psychiatric Serv. 2001:52:482-5.

26. Davidson L, Drake R, Schmutte T, Dinzeo T, Andres-Hyman R. Oil and water or Oil and vinegar? Evidence-based medicine meets recovery. Community Ment HIt J. 2009:45:323.

27. Zimmerman M, Martinez J, Attiullah N, Friedman M, Toba C, Boerescu DA. Why do some depressed outpatients who are not in remission according to the Hamilton Depression Rating Scale nonetheless consider themselves to be in remission? Depress Anxiety. 2012;29:891-5.

28. Corrigan PW, Salzer M, Ralph RO, Sangster Y, Keck L. Examining the factor structure of the recovery assessment scale. Schiz Bull. 2004:30:1035-41.

29. Gladis MM, Gosch EA, Dishuk NM, Crits-Christoph P. Quality of life: expanding the scope of clinical significance. J Consult Clin Psychol. 1999;67: 320-31

30. Trivedi MH, Rush AJ, Wisniewski SR, Warden D, McKinney W, Downing M, Berman SR, Farabaugh A, Luther JF, Nierenberg AA. Factors associated with health-related quality of life among outpatients with major depressive disorder: a STAR* D report. J Clin Psychiat. 2006;67:1-478.

31. Endicott J, Nee J, Harrison W, Blumenthal R. Quality of life enjoyment and satisfaction questionnaire: a new measure. Psychopharmacol Bull. 1993;29:321-6.

32. Hope ML, Hooke GR, Page AC. The value of adding quality of life measures to assessments of outcomes in mental health. Qual Life Res. 2009;18:647-55.

33. ACHS. Clinical indicator user manual: mental health inpatient version 6 . Melbourne: ACHS; 2012

34. Hodgson RE, Lewis M, Boardman AP. Prediction of readmission to acute psychiatric units. Soc Psych Psych Epid. 2001;36:304.

35. Lyons JS, O'Mahoney MT, Miller SI, Neme J, Kabat J, Miller F. Predicting readmission to the psychiatric hospital in a managed care environment: Implications for quality indicators. Am J Psychiat. 1997;154:337-40.

36. Callaly T, Trauer T, Hyland M, Coombs T, Berk M. An examination of risk factors for readmission to acute adult mental health services within 28 days of discharge in the Australian setting. Austral Psychiat. 2011;19:221-5.

37. Byrne SL, Hooke GR, Page AC. Readmission: a useful indicator of the quality of inpatient psychiatric care. J Affect Disorders. 2011;126:206-13.

38. Byrne SL, Hooke GR, Page AC. Readmission to psychiatric hospital following treatment for depression with electroconvulsive therapy: the effect of planned readmissions. J ECT. 2012;28:e12-3. 
39. Corrigan PW, Giffort D, Rashid F, Leary M, Okeke I. Recovery as a psychological construct. Community Ment Health J. 1999;35:231-9.

40. Hancock N, Bund A, Honey A, James G, Tamsett S. Improving measurement properties of the recovery assessment scale with rasch analysis. Am J Occ Ther. 2011;65:77-85.

41. Sklar M, Groessl E, O'Connell M, Davidson L, Aarons GA. Instruments for measuring mental health recovery: a systematic review. Clin Psychol Rev. 2013;33:1082-95.

42. Burgess P, Pirkis J, Coombs T, Rosen A. Assessing the value of existing recovery measures for routine use in Australian mental health services. Aust NZ J Psychiat. 2011;45:267-80.

43. Wing J, Beevor AS, Curtis RH, Park SBG, Hadden S, Burns A. Health of the Nation Outcome Scales (HoNOS): research and development. Br J Psychiat. 1998;172:11-8.

44. Newnham EA, Harwood KE, Page AC. The subscale structure and clinical utility of the health of the nation outcome scale. J Ment Health. 2009:18:326-34.

45. Lovibond PF, Lovibond SH. Self-report scales (DASS) for the differentiation and measurement of depression, anxiety, and stress. Behav Res Ther. 1995;33:335-43.

46. Henry JD, Crawford JR. The short-form version of the Depression Anxiety Stress Scales (DASS-21): construct validity and normative data in a large non-clinical sample. Br J Clin Psychol. 2005;44:227-39.

47. Page AC, Hooke GR, Morrison DM. Psychometric properties of the Depression Anxiety Stress Scales (DASS) in depressed clinical samples. Br J Clin Psychol. 2007:46:283-97.

48. Ronk FR, Korman JR, Hooke GR, Page AC. Assessing clinical significance of treatment outcomes using the DASS-21. Psycholl Assess. 2013;25:1103-10.

49. Ritsner M, Kurs R, Kostizky H, Ponizovsky A, Modai I. Subjective quality of life in severely mentally ill patients: a comparison of two instruments. Qual Life Res. 2002;11:553.

50. Hsu LM. On the identification of clinically significant client changes: reinterpretation of Jacobson's cut scores. J Psychopathol Behav Assess. 1996:18:371-85

51. Cohen J. Statistical power analysis for the behavioral sciences. New Jersey: Lawrence Erlbaum Associates; 1988

52. Rollnick S, Miller WR, Butler C. Motivational interviewing in health care: helping patients change behavior. New York: Guilford Press; 2008.

53. Lambert MJ, Whipple JL, Vermeersch DA, Smart DW, Hawkins EJ, Nielsen SL, Goates M. Enhancing psychotherapy outcomes via providing feedback on client progress: a replication. Clin Psychol Psychother. 2002;9:91-103.

54. Dyer $\mathrm{K}$, Hooke GR, Page AC. Effects of providing domain specific progress monitoring and feedback to therapists and patients on outcome. Psychoth Res. 2016;26:297-306. doi:10.1080/10503307.2014.983207.

55. Shimokawa K, Lambert MJ, Smart DW. Enhancing treatment outcome of patients at risk of treatment failure: meta-analytic and mega-analytic review of a psychotherapy quality assurance system. J Consult Clin Psychol. 2011;78:298-311.

56. Restifo E, Kashyap S, Hooke GR, Page AC. Daily monitoring of temporal trajectories of suicidal ideation predict self-injury: a novel application of patient progress monitoring. Psychother Res. 2015;25:705-13.

57. Atkinson $\mathrm{M}$, Zibin $\mathrm{S}$, Chuang $\mathrm{H}$. Characterizing quality of life among patients with chronic mental illness: a critical examination of the self-report methodology. Am J Psychiat. 1997:154:99-105.

\section{Submit your next manuscript to BioMed Central and we will help you at every step:}

- We accept pre-submission inquiries

- Our selector tool helps you to find the most relevant journal

- We provide round the clock customer support

- Convenient online submission

- Thorough peer review

- Inclusion in PubMed and all major indexing services

- Maximum visibility for your research

Submit your manuscript at www.biomedcentral.com/submit

CBiomed Central 